\title{
Neutronic developments on TOSCA and VESPA: Progress to date
}

\author{
M. Zanetti ${ }^{\mathrm{a}, \mathrm{b}, *}$, S. Bellissima ${ }^{\mathrm{c}}$, L. del Rosso ${ }^{\mathrm{c}}$, F. Masi ${ }^{\mathrm{d}, \mathrm{b}}$, M. Chowdhury ${ }^{\mathrm{b}}$, A. De Bonis ${ }^{\mathrm{b}}$, \\ L. Di Fresco, ${ }^{e, b}$, C. Scatigno ${ }^{e, b}$, J. Armstrong ${ }^{\mathrm{b}}$, S. Rudić ${ }^{\mathrm{b}}$, S.F. Parker ${ }^{\mathrm{b}}$, M. Hartl ${ }^{\mathrm{f}}$, D. Colognesi ${ }^{\mathrm{c}}$, \\ R. Senesi ${ }^{\mathrm{e}, \mathrm{g}}$, C. Andreani ${ }^{\mathrm{e}, g}$, G. Gorini ${ }^{\mathrm{d}, \mathrm{h}}$, F. Fernandez-Alonso ${ }^{\mathrm{b}, \mathrm{i}}$ \\ ${ }^{\text {a }}$ Consiglio Nazionale delle Ricerche, Dipartimento di Scienze Fisiche e Tecnologie della Materia, Roma, Italy \\ ${ }^{\mathrm{b}}$ ISIS Facility, STFC Rutherford Appleton Laboratory, Chilton, Didcot, Oxfordshire OX11 OQX, United Kingdom \\ ${ }^{\mathrm{c}}$ Istituto di Fisica Applicata "Nello Carrara", CNR-IFAC, Sesto Fiorentino, FI, Italy \\ 'Università degli Studi di Milano-Bicocca, Piazza della Scienza 3, 20126 Milano, Italy \\ ' Università degli Studi di Roma "Tor Vergata", Dipartimento di Fisica e Centro NAST, Via della Ricerca Scientifica 1, Roma, 00133, Italy \\ ${ }^{\mathrm{f}}$ European Spallation Source ERIC, Box 176, S-221 00 Lund, Sweden \\ ${ }^{g}$ CNR-IPCF, Sezione di Messina, Messina, Italy \\ ${ }^{\mathrm{h}}$ Istituto di Fisica del Plasma "P. Caldirola", CNR-IFP, Milano, Italy \\ ${ }^{\mathrm{i}}$ Department of Physics and Astronomy, University College London, Gower Street, London WC1E 6BT, United Kingdom
}

\section{A R T I C L E I N F O}

\section{Keywords:}

TOSCA

VESPA

Neutron instrument

Vibrational spectrometer

\begin{abstract}
A B S T R A C T
We report recent developments regarding the TOSCA (ISIS, UK) and VESPA (ESS, Sweden) neutron broadband chemical spectrometers, both joint ventures between CNR (IT) and ISIS (UK). TOSCA has seen the first major upgrade since it first became operational over fifteen years ago. The new design of the primary spectrometer, which exploits a state-of-the-art, high- $m$ neutron guide and associated chopper system, is boosting the useful neutron flux by over an order of magnitude. Feasibility studies for an upgrade of the secondary spectrometer have been performed, outlining an additional order-of-magnitude gain in performance. In the case of VESPA, the novel characteristics and challenges arising from a long-pulse spallation source such as ESS are part of the drivers of the instrument design. For both the primary and secondary spectrometers, a detailed analysis of expected performance, supported by both simulations and analytical models, is being carried out, also capitalizing from experience on TOSCA. Indeed, for instrument design and optimization, extensive neutron-transport simulations and baseline studies of neutronic response have become a must, along with extensive benchmarking against much-needed experimental data. All these combined efforts represent the first opportunity to benchmark a broadband, high-resolution chemical spectrometer in terms of measured vs. simulated response.
\end{abstract}

\section{High-resolution broadband chemical spectrometers}

Inelastic Neutron Scattering (INS) as a vibrational spectroscopy technique [1] is widely used across chemistry, materials science, biology, and beyond. The workhorses for this kind of studies can be called "High-resolution Broadband Chemical Spectrometers". Here, we focus on time-of-flight instruments at pulsed spallation sources in socalled indirect geometry, and whereby final-energy selection is attained with a crystal after scattering by the sample. With them, good-quality spectra (i.e. high count rate and resolution) can be obtained with reasonably small quantities of specimen and easy operation. This type of instruments is generally divided into a Primary Spectrometer (PS), dedicated to neutron delivery from a moderator to the sample, and a Secondary Spectrometer (SS) which processes neutrons scattered from the sample and delivers them to the detectors.

In the PS the polychromatic beam, coming from the moderator, is generally worked on by a chopper system (i.e. shaping or cleaning it) and transported onto the sample by a neutron delivery device (e.g. an evacuated tube or a super-mirror guide). The SS is arranged in such a way that the final energy of neutrons that can be detected after scattering by the sample is fixed. This is achieved via the use of an analyser crystal, typically HOPG (Highly Oriented Pyrolytic Graphite), which selects the neutrons through Bragg scattering. In order to eliminate higher-order crystal reflections, a low-pass energy filter is used. The filter is typically made with a thick block of beryllium, selecting the bandwidth by exploiting the Bragg cut-off at $5.1 \mathrm{meV}$, which is sliced with neutron-absorbing materials [2] and cooled at least to liquid-nitrogen temperatures in order to reduce spurious scattering. Finally,

\footnotetext{
* Corresponding author. Consiglio Nazionale delle Ricerche, Dipartimento di Scienze Fisiche e Tecnologie della Materia, Roma, Italy.

E-mail address: matteo.zanetti@amministrazione.cnr.it (M. Zanetti).
} 
detector banks are placed on the path of the analysed neutrons. This typically involves the adoption of ${ }^{3} \mathrm{He}$ detector tubes, which combine high efficiency at low neutron energies with a robust background-rejection capability via a low sensitivity to high-energy neutrons and gammas.

We recall that the position and the orientation of both the analysers and the detectors are strongly coupled. When high-resolution is required for high energy transfers, only a careful design allows a dramatic reduction in the width of the neutron time-of-flight distribution in the SS (time-focusing). This can be achieved, for instance, by keeping the sample, analyser and detector planes parallel. On the other hand, highresolution at low energy transfers, and especially in the elastic region of the spectrum, is achieved by reducing the width of the energy distribution of secondary neutrons accepted by a selected detector (energy-focusing). Keeping this in mind, the crystals are then arranged so as to cover the largest possible solid angle while maintaining time-focusing, and keeping the Bragg angles of the analysers within a range that does not imply selection of neutrons incompatible with the filter cut-off.

A set of diffraction detectors, even if only with basic capabilities, is generally included in the spectrometer to increase versatility and to allow performing concurrent dynamic and structure experiments. This is not a prerogative of this kind of spectrometer, but Quasielastic and Deep-Inelastic instruments, like OSIRIS (ISIS, UK) [3] and VESUVIO (ISIS, UK) [4], are also equipped with this kind of complementary equipment.

This contribution focuses on recent developments on two of these spectrometers, namely, TOSCA (ISIS, UK) [5] and VESPA (ESS, Sweden) [6], both joint ventures between CNR (IT) and ISIS (UK).

\section{TOSCA}

In the above context, the first thought goes to the TOSCA spectrometer, which has been the gold standard for INS in the last two decades, as demonstrated by the TOSCA INS database [7] and the recent International Review [8]. Since it succeeded TFXA (Time Focused Crystal Analyser) in 1998, TOSCA underwent a number of small upgrades: first a rearrangement of the scattering banks, in order to collect both backscattered and forward-scattered neutrons, as well as an increase of distance from the moderator to the current $17 \mathrm{~m}$, which enhanced the energy resolution; the improvement of the beryllium filters; and finally the ability of exploiting the empty pulse resulting from ISIS TS2 operation, thus increasing the energy transfer range [2].

The first major upgrade of TOSCA (Fig. 1), instead, is quite recent, and was completed in the first months of 2017 [9]. A new PS, which exploits a state-of-the-art, high- $m$ neutron guide, has been provided to the instrument in order to dramatically increase the number of neutrons delivered to the sample. The upgrade was guided by a combination of simulation (performed with the McStas package [10]) and experimental characterization of the instrument, which also allows us to quantify the neutronic response before and after the upgrade. A number of preliminary simulations set the achievable gain from the guide installation, then allowing a refined design [11] which ended in the currently installed tapered guide, characterized by $m$-values that, moving towards sample position, increase from 5 to 7 [9]. The simulations were supported by a careful characterization of the beam [12], then repeated after the upgrade [9] which allows us to identify an average gain of more than a factor ten over the most useful range of the spectrum, with a maximum of two orders of magnitude in flux at the lowest incident energies, and no detriment to resolution or signal-to-noise ratio. A similar procedure is being followed for the next possible upgrade of the SS. From the point of view of neutronic calculations, the feasibility of an order-of-magnitude increase in performance, via the installation of large parabolic analysers, has been assessed [13], while a detailed characterization of the current SS is being carried out.

\section{VESPA}

VESPA will be the ESS high-resolution broadband chemical spectrometer. The joint efforts of CNR (IT) and ISIS (UK) to develop this new instrument started a few years ago and lately have led to the detailed design phase. For VESPA, the current design of both its PS and SS is being guided by a detailed analysis of expected performance, supported by both simulations and analytical models, capitalizing on experience gained with TOSCA. The new spectrometer is designed so as to exploit the long pulse produced by the ESS neutron source by means of the Wavelength Frame Multiplication concept [14].

The PS comprises of a chopper system to select the appropriate neutrons from the ESS long pulse and a high- $m$ guide to deliver them to the sample. The chopper system is based on three Pulse Shaping Choppers (PSC), in optical blind configuration [15], placed relatively near the moderator, which allow the flexibility to trade flux for energy resolution by setting two different operational modes: High-Resolution (HR) and Low-Resolution (LR), characterized by a $1 \%$ and $2 \%$ almostconstant relative energy resolution, respectively. The long pulse is shaped into three frames with $3.7-9 \mathrm{meV}, 8-28 \mathrm{meV}$, and $23-500 \mathrm{meV}$ incident energy ranges. The chopper system is also equipped with a bandwidth chopper and a sub-frame overlap chopper, that help to keep the three frames produced by the PSC's well separated in time.

Neutrons coming from the moderator are initially collected by an elliptically-shaped feeder placed in the monolith, pass through the PSC assembly, and are then transported by a $51 \mathrm{~m}$ long elliptical guide (Fig. 2), whose geometry has been optimized with guide_bot [16]. The optimization of the guide shape involved setting a number of crosssection constraints at several positions along the beamline and maximum divergence of $2^{\circ}$ at the sample position with an adequate beam homogeneity. Before this final optimization, a set of guides with different geometries, which were chosen by performing initial analytical calculations, was developed. These were then tested with McStas and a baseline configuration was identified. The final optimization step using guide_bot aimed at increasing the flux at the sample position across the fingerprint region with respect to the baseline. With $12.6 \mathrm{~m}^{2}$ of supermirror surface, the optimized guide covers most of the $59 \mathrm{~m}$ flight-path between the moderator and the sample, and illuminates

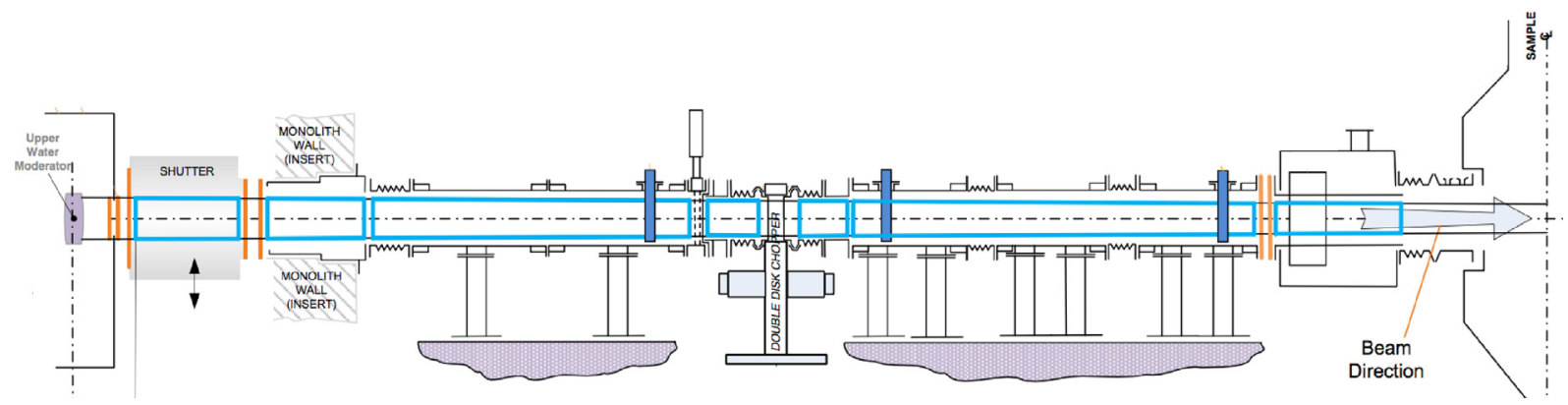

Fig. 1. TOSCA upgrade. The new guide configuration is reported in Ref. [9]. 

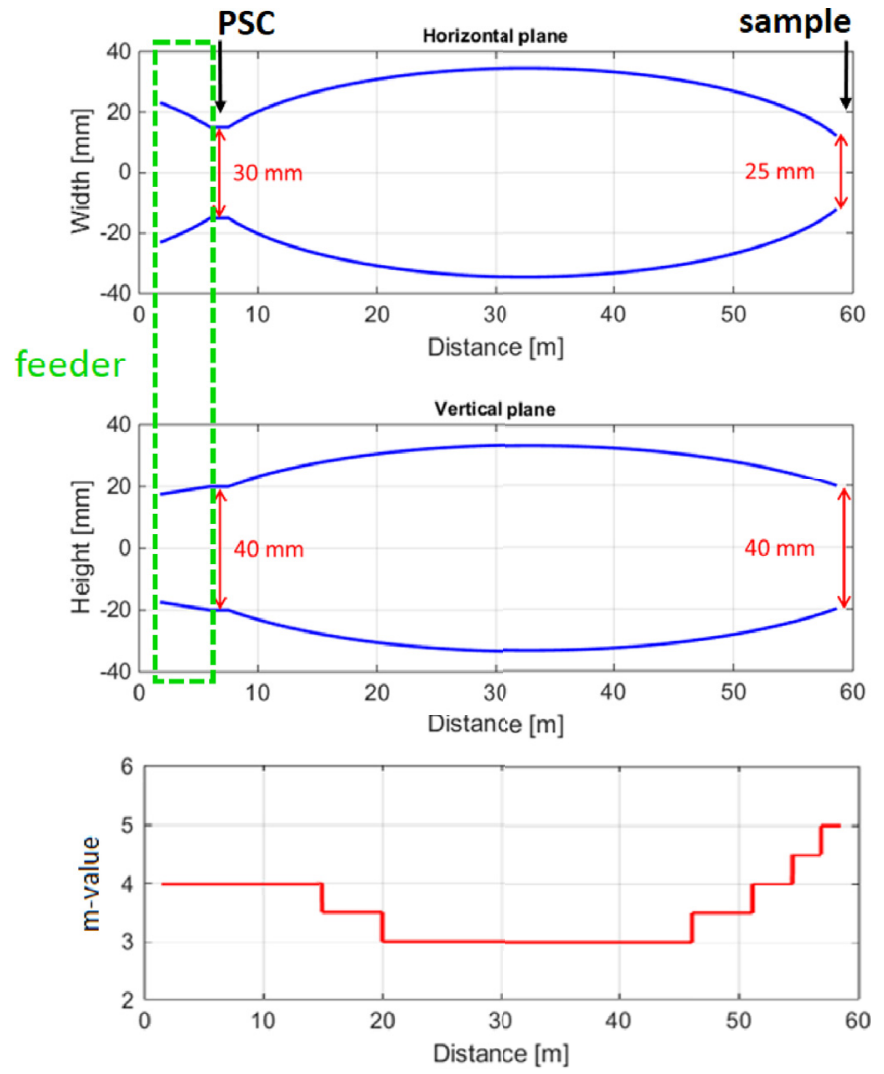

Fig. 2. VESPA guide geometry, fully optimized via the guide_bot software package. The geometry is reported together with the $m$-values selected for the different sections.

homogeneously an area of $25 \times 40 \mathrm{~mm}^{2}$, while viewing the thermal centre of the ESS moderator on the opposite side.

The SS employs elliptically shaped analysers, made with $2^{\circ}$-mosaic$1.2 \times 1.2-\mathrm{cm}^{2}$ HOPG crystal tiles, and designed for energy-focusing and time-focusing, allowing for a total coverage of the sample of about 5.2 steradian. By elliptically shaped we mean that, starting from a generating row in two dimensions, each HOPG tile centre is placed over an elliptical curvature (See Fig. 3(a)). One would expect that the sample and the focusing point of such an arrangement would lie in the ellipse foci. In fact, the tiles are not oriented tangent to the ellipse, but each

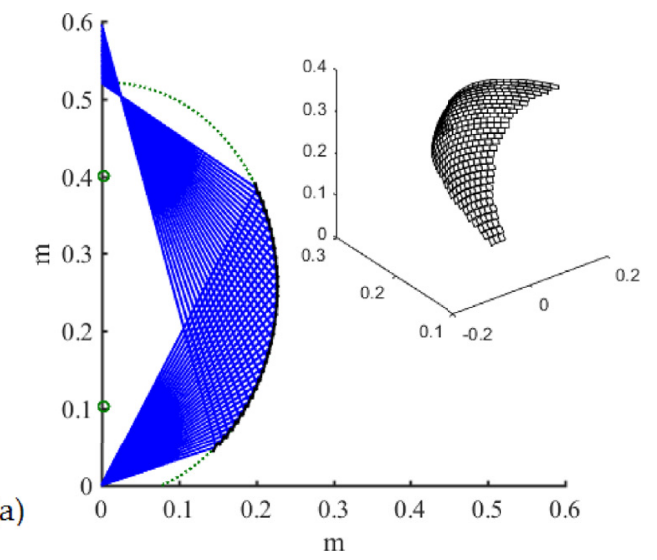

one is tilted toward a different, but unique, point. This new focus point is selected in such a way that the neutrons analysed at the tile centre, thus with a given energy, take a unique time to reach the focus from the sample. This feature allows reducing the width of the secondary neutron time-of-flight distribution, thus allowing for an effective time-focusing. The generating row, so arranged, is then rotated around the sample axis so as to obtain the final analyser geometry (See Fig. 3(a)).

The analyser, selecting final energies comprised between 3.8 and $4.7 \mathrm{meV}$ (See Fig. 3(b)), enables access to the so-called "Elastic Line" with a FWHM of about $250 \mu \mathrm{eV}$. The focused geometry is designed to send the selected neutrons towards a position-sensitive detector bank equipped with ${ }^{3} \mathrm{He}$-tubes. Differently from the current TOSCA design, in which flat analysers parallel to sample and detectors (non-positionsensitive tubes) are used, this kind of arrangement is fundamental in order to recover a well defined time-of-flight distribution in each pixel. Thanks to time-focusing, the average time-of-flight in the SS is around $730 \mu$ s, with a standard deviation below $10 \mu$ s for each pixel (See Fig. 3(b)). This temporal width is a threshold carefully tuned to PSC operation in order to provide an optimal match with the time structure delivered to the sample, that allows us to obtain the unprecedented $1 \%$ relative energy resolution from $50 \mathrm{meV}$ up to $500 \mathrm{meV}$. The shape of the beryllium filter and its slicing are designed according to the geometry of the analysers, following the trajectories of the reflected neutrons toward the detectors.

Finally, the scientific scope of VESPA is enhanced by diffraction capabilities over a Q-range of $1.8-31 \AA^{-1}$, thus the instrument will be able to collect simultaneous dynamic and structural data, which is particularly useful to identify phase changes during in situ and operando experiments, or perform structure refinement, for example.

The VESPA spectrometer will cover a broad range of energy transfers (up to $500 \mathrm{meV}$ ) in one shot, making kinetic or parametric experiments feasible and easy to be interpreted. Thanks to the high resolution and flux in the energy range of interest (i.e. vibrational fingerprint spectral region between 60 and $220 \mathrm{meV}$ ), VESPA will produce world-leading science in INS vibrational spectroscopy. Moreover, VESPA will have access to the "Elastic Line", thus allowing to get enough information to perform preliminary QENS dynamics studies.

\section{Instrument simulation}

A detailed neutronic design of the TOSCA upgrades and of VESPA would not be possible if simulation packages of INS instruments, like McStas [10], were not available. Or, to be specific, a detailed, optimized design, which does not rely on a number of hard-to-test assumptions as
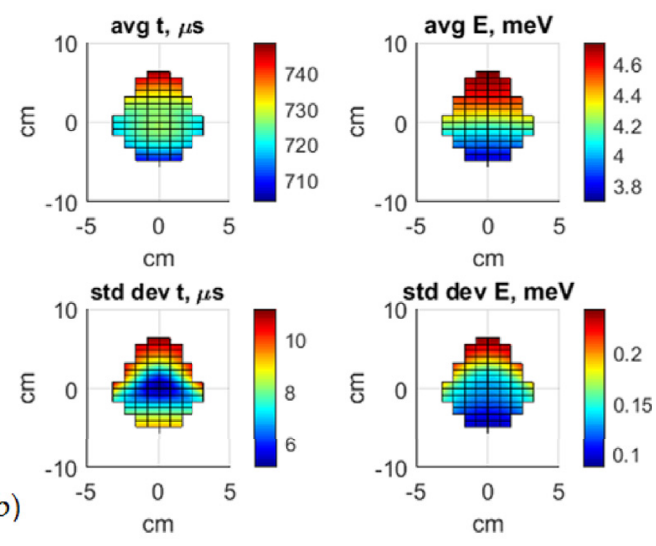

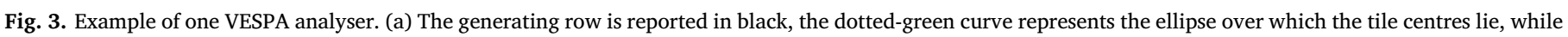

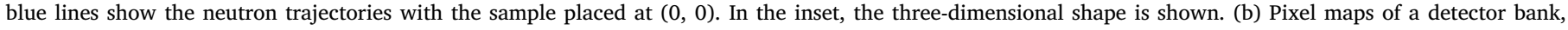

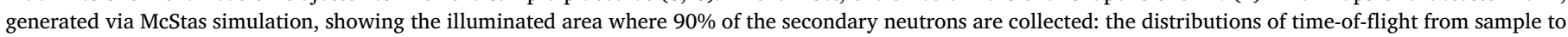

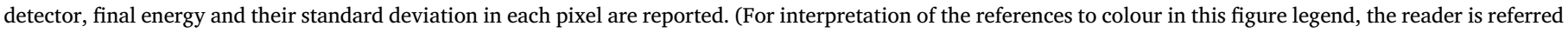
to the Web version of this article.) 


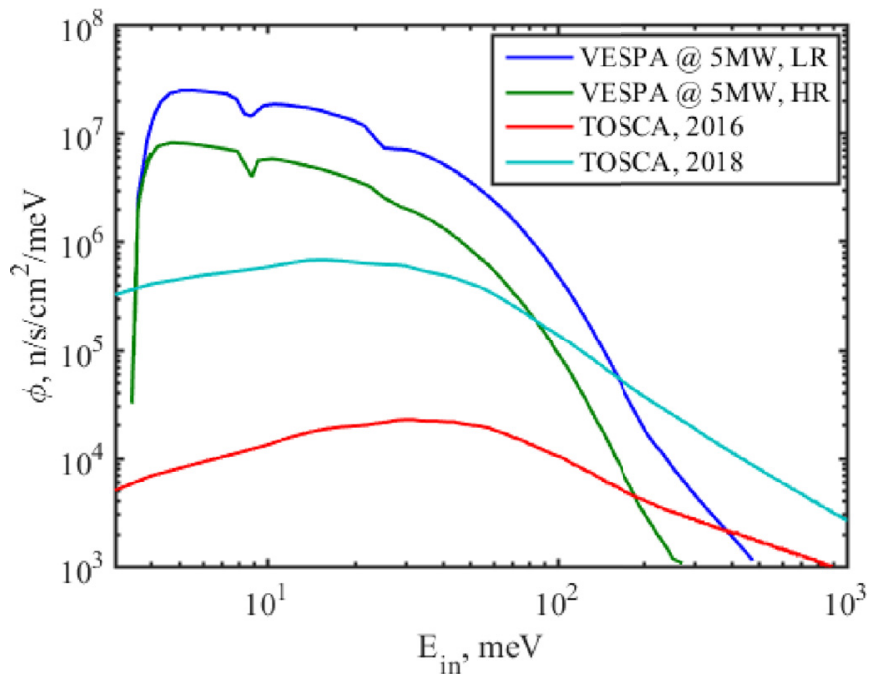

Fig. 4. Simulated flux at sample position. In order to allow a reasonable comparison between the two instruments, only simulation data are reported. For TOSCA, the flux at the sample position before the upgrade (as in the year 2016) and after the upgrade (as in the year 2018) is shown. For VESPA, the flux at the sample position for the LR and HR modes is shown.

it was done in the past would not be achievable. Instead of relying on analytical models, which involve plenty of idealisation, McStas allows detailed analysis of departures from ideal conditions (e.g. time-focusing and its effect on resolution). This situation is particularly true when the many different contributions to the neutronic response that are associated with both the PS and the SS are considered in detail, like when evaluating for the trade-off between flux and spectral resolution, or the effects related to complex geometries.

Extensive simulation efforts have been made using the McStas package. For instance, a first kind of evaluation is the efficiency of the PS, which can be evaluated by checking the expected flux at the sample position. This is reported in Fig. 4 for both TOSCA and VESPA. The gain achieved with the TOSCA guide upgrade is evident, while for VESPA the trade-off between intensity and resolution is shown. The resolution performances and their effect on a typical sample spectrum is shown in Figs. 5 and 6, respectively.

In particular, in Fig. 5 the relative FWHM for the two instruments is reported. From the simulation point of view, a synthetic " $\delta$ - sample", with extremely narrow spectral features at a given energy transfer, was developed and used to study the peak shapes. The FWHM calculated assuming that the peaks are Gaussian are then compared with data collected on TOSCA [2] with a 2,5-diiodothiophene calibrant. In this case, either the intrinsic width of spectral features or the presence of several features over a narrow range leading to congestion affect the shape of the collected peaks and lead to resolution values which can be different from the expected theoretical ones.

In Fig. 6 the simulation of a typical calibration sample for INS, namely low-temperature zirconium(II) hydride, $\mathrm{ZrH}_{2}$, is reported along with the corresponding experimental data recently collected on TOSCA. All the calculated $\mathrm{ZrH}_{2}$ spectra have been obtained starting from the $\mathrm{H}$ projected Vibrational Density of States (H-VDOS) in conjunction with the well-known Gaussian approximation, which is used to transform the $\mathrm{H}$-VDOS into the instrumental self-scattering law, $\mathrm{S}_{s}(\mathrm{Q}, \omega)$, evaluated along the appropriate $(\mathrm{Q}, \omega)$ kinematic path and including energy resolution effects. As for the H-VDOS, a synthetic model has been set up after an extensive review of the published literature on this subject (partially reported in Refs. $[17,18]$ ). The comparison between experimental data, gathered on TOSCA, and simulation demonstrates the adequacy of the models employed. Likewise, the effect of changing the resolution on VESPA is also apparent.

For VESPA, additional efforts for implementing background

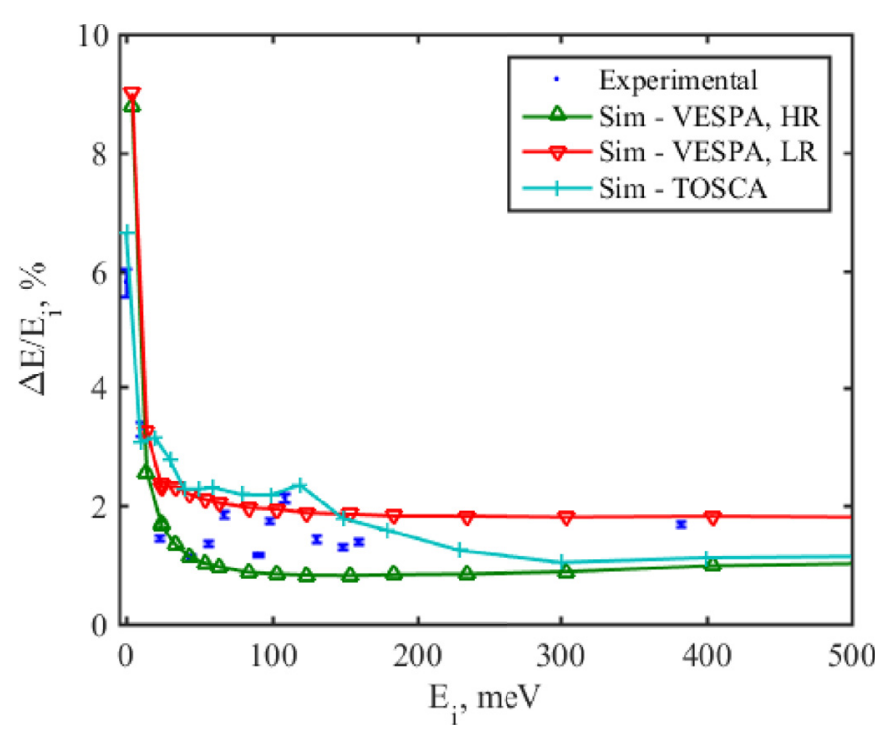

Fig. 5. Simulated resolution, compared with data from TOSCA [2]. Continuous curves represent the expected resolution from simulation of the " $\delta$ - sample", while the FWHM of selected peaks from the 2,5-diiodothiophene calibrant sample is represented by blue circles. For TOSCA, the "bulky feature" between 50 and $200 \mathrm{meV}$ is well represented, and is due to the width of the neutron pulse. For VESPA, the nearly constant resolution in the LR and HR modes is shown. (For interpretation of the references to colour in this figure legend, the reader is referred to the Web version of this article.)

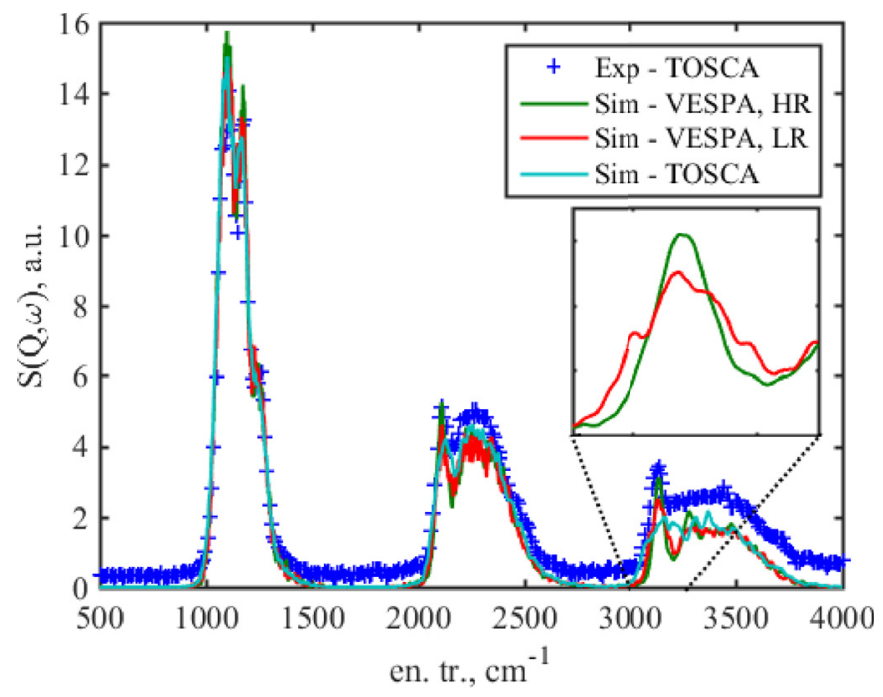

Fig. 6. Simulated sample of $\mathrm{ZrH}_{2}$, compared with data obtained through the Xpress route on TOSCA [21]. The inset shows the effect of changing the resolution mode on VESPA.

reduction, through a specific and innovative design of the "shielding", both in the PS and in the experimental cave, is being performed with detailed neutron transport simulations, using state-of-the art codes, such as McStas [10] and MCNP-X [19].

Advances in simulation tools also allow integrating scientific and engineering efforts. For instance, the VESPA analysers have been designed with tight iterations between the neutronics and mechanical studies, by importing the realistic geometry, as defined with CAD, directly into McStas, gaining immediate insights on design refinements and allowing a real optimization of these devices. A further benefit from this approach is the optimization of the computational side of the simulations. We have developed a method in which, according to the grid defined by the imported CAD surface, the tile position is dynamically assigned for each neutron scattered from the sample toward the 
analysers. The remaining neutrons that would be otherwise lost are suppressed, clearing space for the following "neutron history".

\section{Outlook}

To optimize instrument design, extensive neutron-transport simulations and baseline studies of neutronic response have become a must, along with extensive benchmarking against much-needed experimental data. This combined approach was not the norm in the past (i.e. for TFXA or the earlier TOSCA), but has now become an essential ingredient in recent and forthcoming instrument upgrades on TOSCA, or in the design of VESPA.

A good practice has been established during the TOSCA upgrade: combining preliminary simulations [11] with detailed characterization [12]. Similar plans are being implemented both for VESPA design and TOSCA secondary spectrometer upgrade, characterizing the neutronic response of the different materials and components that will be used in the final instruments, and validating the models used to design them. For instance, some of the materials to be used in the instruments are being tested, by studying their neutron transmission properties, as recently demonstrated on VESUVIO [20].

Furthermore, as we progress in the model validation and in the understanding of the different causes to the neutronic response, more detailed models are being developed, which can include effects that previously were not considered (e.g. the effect of scattering within the beryllium filter), and are incrementally added to the complete virtual instruments. This work represents the first opportunity to benchmark a high-resolution broadband chemical spectrometer in terms of measured vs. simulated response.

\section{Acknowledgements}

The authors gratefully acknowledge the Science \& Technology Facilities Council (STFC - UK Research and Innovation), the computing resources from the STFC Scientific Computing Department's SCARF cluster, and the CNR, within the CNR-STFC Agreement (2014-2020) No. 3420 and the ESS-ERIC for Instrument VESPA Phase 1. The authors are also immensely grateful to all the engineers who have made possible the TOSCA upgrade and the progress of VESPA to the new design phase.

\section{References}

[1] Philip C.H. Mitchell, Stewart F. Parker, Anibal J. Ramirez-Cuesta, John Tomkinson, Vibrational Spectroscopy with Neutrons, World Scientific, 2005, https://doi.org/ 10.1142/5628 http://dx.doi.org/10.1142/5628.

[2] S.F. Parker, F. Fernandez-Alonso, A.J. Ramirez-Cuesta, J. Tomkinson, S. Rudić, R.S. Pinna, G. Gorini, J.F. Castañon, Recent and future developments on TOSCA at ISIS, J. Phys. Conf. Ser. 554 (1) (2014) 012003 http://stacks.iop.org/1742-6596/ $554 / \mathrm{i}=1 / \mathrm{a}=012003$.

[3] F. Demmel, D. McPhail, C. French, D. Maxwell, S. Harrison, J. Boxall, N. Rhodes, S. Mukhopadhyay, I. Silverwood, V.G. Sakai, F. Fernandez-Alonso, ToF- backscattering spectroscopy at the ISIS facility: status and perspectives, J. Phys. Conf. Ser. 1021 (1) (2018) 012027 http://stacks.iop.org/1742-6596/1021/i=1 $\mathrm{a}=012027$.

[4] G. Romanelli, M. Krzystyniak, R. Senesi, D. Raspino, J. Boxall, D. Pooley, S. Moorby, E. Schooneveld, N.J. Rhodes, C. Andreani, F. Fernandez-Alonso, Characterisation of the incident beam and current diffraction capabilities on the VESUVIO spectrometer, Meas. Sci. Technol. 28 (9) (2017) 095501 http://stacks.iop.org/0957-0233/ $28 / \mathrm{i}=9 / \mathrm{a}=095501$.

[5] Accessed on 2018-08-28, https://www.isis.stfc.ac.uk/Pages/Tosca.aspx.

[6] A. Fedrigo, D. Colognesi, M. Bertelsen, M. Hartl, K. Lefmann, P.P. Deen, M. Strobl, F. Grazzi, M. Zoppi, VESPA: the vibrational spectrometer for the European spallation source, Rev. Sci. Instrum. 87 (6) (2016) 065101, https://doi.org/10.1063/1. 4952430 http://dx.doi.org/10.1063/1.4952430.

[7] Accessed on 2018-08-28, https://www.isis.stfc.ac.uk/Pages/INS-database.aspx.

[8] S. Rudić, A.J. Ramirez-Cuesta, S.F. Parker, F. Fernandez-Alonso, R.S. Pinna, G. Gorini, C.G. Salzmann, S.E. McLain, N.T. Skipper, TOSCA International Beamline Review, RAL Technical Reports RAL-TR-2013-015 STFC, 2013, http://purl.org/net/ epubs/work/11216706.

[9] R.S. Pinna, S. Rudić, S.F. Parker, J. Armstrong, M. Zanetti, G. Škoro, S.P. Waller, D. Zacek, C.A. Smith, M.J. Capstick, D.J. McPhail, D.E. Pooley, G.D. Howells, G. Gorini, F. Fernandez-Alonso, The neutron guide upgrade of the TOSCA spectrometer, Nucl. Instrum. Methods Phys. Res. Sect. A Accel. Spectrom. Detect. Assoc. Equip. 896 (2018) 68-74 https://doi.org/10.1016/j.nima.2018.04.009.

[10] P. Willendrup, E. Farhi, K. Lefmann, McStas 1.7 - a new version of the flexible Monte Carlo neutron scattering package, Phys. B Condens. Matter 350 (1, Supplement) (2004) E735-E737 proceedings of the Third European Conference on Neutron Scattering https://doi.org/10.1016/j.physb.2004.03.193.

[11] R.S. Pinna, S. Rudić, S.F. Parker, G. Gorini, F. Fernandez-Alonso, Monte Carlo simulations of the TOSCA spectrometer: assessment of current performance and future upgrades, EPJ Web Conf. 83 (2015) 03013 http://dx.doi.org/10.1051/ epjconf/20158303013.

[12] R.S. Pinna, S. Rudić, M.J. Capstick, D.J. McPhail, D.E. Pooley, G.D. Howells, G. Gorini, F. Fernandez-Alonso, Detailed characterisation of the incident neutron beam on the TOSCA spectrometer, Nucl. Instrum. Methods Phys. Res. Sect. A Accel. Spectrom. Detect. Assoc. Equip. 870 (2017) 79-83 https://doi.org/10.1016/j.nima. 2017.07.018.

[13] R.S. Pinna, S. Rudić, M. Zanetti, D. Zacek, S.F. Parker, G. Gorini, F. FernandezAlonso, Monte Carlo Simulations for the TOSCA Secondary Spectrometer Upgrade, RAL Technical Reports RAL-TR-2017-013 STFC, 2017, http://purl.org/net/epubs/ work/35959990.

[14] F. Mezei, M. Russina, Advances in neutron scattering instrumentation, in: I. Anderson, B. Guerard (Eds.), Proceedings of SPIE, vol. 4785, 2002, p. 22.

[15] A. van Well, Double-disk chopper for neutron time-of-flight experiments, Phys. B Condens. Matter 180-181 (1992) 959-961 https://doi.org/10.1016/09214526(92)90521-S.

[16] M. Bertelsen, The automatic neutron guide optimizer guide_bot, Nucl. Instrum. Methods Phys. Res. Sect. A Accel. Spectrom. Detect. Assoc. Equip. 867 (2017) 195-203 https://doi.org/10.1016/j.nima.2017.06.012.

[17] A.C. Evans, D.N. Timms, J. Mayers, S.M. Bennington, Neutron-scattering study of the impulse approximation in ZrH2, Phys. Rev. B 53 (1996) 3023-3031 http://dx. doi.org10.1103/PhysRevB.53.3023.

[18] W. Zheng, R.G. McClarren, Emulation-based calibration for parameters in parameterized phonon spectrum of ZrHx in TRIGA reactor simulations, Nucl. Sci. Eng. 183 (1) (2016) 78-95 http://dx.doi.org/10.13182/NSE15-48.

[19] D.B. Pelowitz, MCNPX User's Manual, Version 2.7.0, Report LA-CP-11-00438 Los Alamos National Laboratory, 2011.

[20] G. Romanelli, S. Rudić, M. Zanetti, C. Andreani, F. Fernandez-Alonso, G. Gorini, M. Krzystyniak, G. Škoro, Measurement of the para-hydrogen concentration in the ISIS moderators using neutron transmission and thermal conductivity, Nucl. Instrum. Methods Phys. Res. 888 (2018) 88-95 http://dx.doi.org/10.1016/j.nima. 2018.01.039.

[21] M. Zanetti, S. Rudić, Xpress 1790145, STFC ISIS Facility, (2017) https://doi.org/10. 5286/ISIS.E.RB1790145-1. 